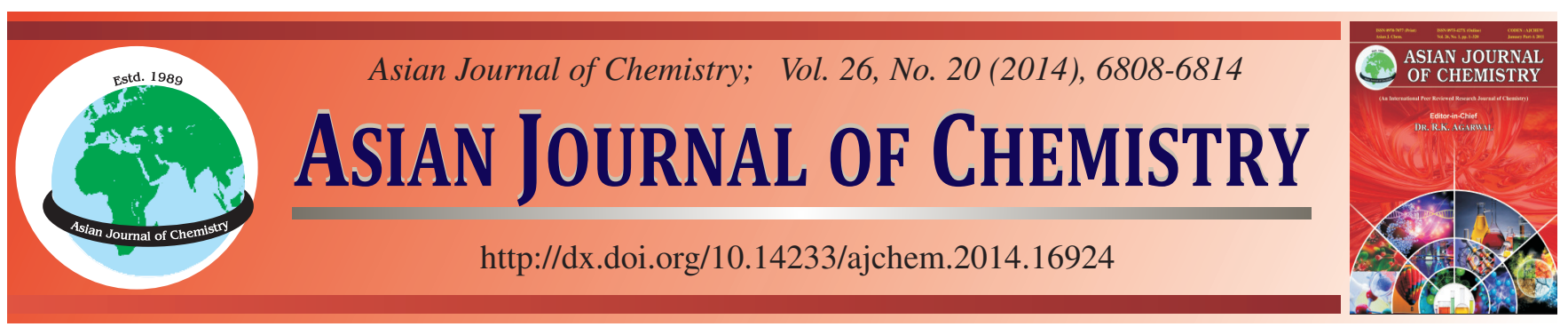

\title{
Variant Molar Mass and Concentration Effect of Polyethylene Glycol over the Physico-Chemical Behaviour of CTAB and SDS
}

\author{
Hazrat Ali ${ }^{1}$, Azim Khan ${ }^{1}$, Irfan Ullah ${ }^{2, *}$, Gulrez Fatima Durrani, \\ Musa Kaleem Baloch ${ }^{2}$, Abdur Rauf ${ }^{3}$, Ayesha Niazi ${ }^{1}$ and Muhammad Akram ${ }^{4}$
}

\begin{abstract}
${ }^{1}$ Department of Chemistry, Gomal University, Dera Ismail Khan-29050, Pakistan
${ }^{2}$ Department of Chemistry, University of Sargodha Sub-Campus Bhakkar-30000, Pakistan

${ }^{3}$ Department of Chemistry, University of Science and Technology, Bannu-28100, Pakistan

${ }^{4}$ Medicinal Botanic Center, PCSIR Labs Complex, Peshawar-25120, Pakistan
\end{abstract}

*Corresponding author: E-mail: smileplease79@gmail.com

\begin{abstract}
Ionic surfactant such as sodium dodecylsulfate (SDS) and N-cetyl N,N,N-trimethyl ammonium bromide (CTAB) has been tensiometrically and conductometrically studied in the presence and absence of water soluble polymer i.e., varying concentration and molar mass of poly(ethylene glycol). Surface tension study shows that poly(ethylene glycol) (PEG) is surface active and its mixture with CTAB and SDS effect the $\mathrm{CMC}$ of these surfactants i.e., CMC of CTAB increases with addition of PEG, changes more when concentration or molar mass increases while the effect is opposite for SDS. The surface tension curve of the mixed system of CTAB-PEG show that after CMC, CTAB molecules replace all the PEG molecules or its loops while in case of SDS-PEG some molecules or loop of the polymer always been present at the interface beyond CMC of SDS. Thermodynamic parameters calculated for the mixed systems shows that free energy of micellization for CTAB-PEG increases with the increase in concentration and molar mass of polymer, but opposite result was observed for SDS-PEG mixture. The conductometric and tensiometric results, regarding micellization were comparable. Whereas, different micellization behavior of CTAB and SDS in the presence of PEG is due to the hydrophobic interaction and ion dipole interaction between CTAB-PEG and SDS-PEG, respectively.
\end{abstract}

Keywords: Ionic surfactant, Poly(ethylene glycol), Molar mass, Interface, Ion dipole interaction, Hydrophobic interaction.

\section{INTRODUCTION}

The interactions between water-soluble polymers and surfactants have been the subject of academic and industrial research interest for the past decades. Because surfactants are relevant in processes such as detergency, wetting, foaming or emulsification. While polymers are used to control the viscosity of solutions. Therefore, mixing these substances give some synergistic properties as a result of interaction between them. So understanding these interactions has great technological significance in further investigations of the complex structure of polymers and surfactants. Most industrial formulations, such as laundry detergents, personal care products, water-soluble paints and oil recovery fluids, contain both water-soluble polymers and surfactants ${ }^{1-5}$. The mixed systems of the water soluble polymer and ionic surfactants have properties differing from those of the pure components due to the complexation of the polymer with the surfactants ${ }^{6}$. It is well known that ionic surfactants interact much more strongly than non-ionic surfac- tants with neutral polymers ${ }^{3}$ however anionic surfactants have a higher affinity for neutral polymers than cationic surfactants ${ }^{7}$. Ionic surfactants form micellar complexes with the polymer chain, which are formed at the critical aggregation concentration (CAC), which is substantially lower than the critical micellar concentration (CMC) of the pure surfactant solution.

Mixed system of ionic surfactant and neutral polymer have been extensively studied by viscometry ${ }^{8}$ surface tension ${ }^{9,10}$ dialysis ${ }^{11}$ conductivity ${ }^{12,13}$ potentiometric measurements ${ }^{14} \mathrm{NMR}$ spectroscopy ${ }^{15,16}$ light and neutron scattering ${ }^{17}$ electrophoretic light scattering ${ }^{18,19}$ fluorescence spectroscopy ${ }^{20,21}$ and kinetic probes $^{22,23}$. These can be categorized into two main types ${ }^{24}$. The first is weakly interacting systems normally consisting of a neutral polymer with a charged surfactant where the interactions are largely hydrophobic in nature. In these systems, some enhanced adsorption occurs in the presence of both species, but after micellization of the surfactant in the bulk solution, the polymer largely displaced from the interface. The second category consists of strongly interacting systems where 
opposite electrostatic charges or strong hydrophobic interactions can lead to the formation of multilayers of alternating polymer and surfactant layers at liquid interfaces. These generally consist of two to three layers, with a top layer of surfactant followed by a layer of polymer and a second layer of surfactant, but these have been mostly studied in very dilute solutions up to a few times the critical micelle concentration of the surfactant and for polymer concentrations in the ppm range $\mathrm{e}^{24}$.

In this report we investigated the effect of water soluble polymer poly(ethylene glycol) (PEG) over the physio-chemical properties of ionic surfactants (SDS and CTAB) also the effect the different molar mass and concentration of poly(ethylene glycol) have been studied by surface tension and conductance. To evaluate the interactions between the PEG (at 4K, $10 \mathrm{~K}$ and $20 \mathrm{~K}$ ) with ionic surfactants the surface tension measurement and conductance data have presented in way which help to understand the type of interaction.

\section{EXPERIMENTAL}

N-Cetyl N,N,N- trimethyl ammonium bromide (CTAB) was obtained from Fluka (Buchs, Germany), sodium dodecyl sulfate (SDS) was obatained from E. Merck, Germany, being of analytical grade used without further purification. whereas PEG having molecular mass 4, 10 and $20 \mathrm{~kg} / \mathrm{mol}$ was donated by Department of Physical Chemistry, University of Bayreuth, Germany which was originally obtained from Shell (Munich, Germany) and used as such. The deionized water whose conductance ranged from 1.2 to $2.5 \mathrm{lS} / \mathrm{cm}$ was used as a solvent.

Sample preparation: Known concentrations of SDS, CTAB, SDS + PEG, CTAB + PEG and PEG (4, 10, $20 \mathrm{~kg} / \mathrm{mol})$ were prepared in deionized water as a stock solution. Solutions of pure SDS, CTAB and PEG of different concentration were obtained by diluting the stock solution. Mixed stock solutions of SDS + PEG and CTAB + PEG were obtained by diluting the stock solution with PEG solution instead of water.

Surface tension measurement: The surface tension was measured using TE3 LAUDA tensiometer, supplied by LAUDA, Germany. All measurements were made sufficiently slowly so as to ensure equilibrium conditions. The temperature was kept constant at $25 \pm 0.01^{\circ} \mathrm{C}$. For the purpose, Ecoline Circulation Thermostat Model E 015T Germany was employed.

The conductance of the solution of surfactant, polymer and their mixture was measured in de-ionized water using InoLab Cond. 720 conductivity meter (Buchs, Germany). The conductivity cell and the vessel used for the measurement were properly cleaned and the conductivitymeter was standardized before use.

\section{RESULTS AND DISCUSSION}

Determination of critical micelle concentration (CMC) and interfacial adsorption: A number of techniques have been employed to measure amphiphilic character of the surfactant and polymer; among these techniques the surface tension $(\gamma)$ measurement is commonly used method. Critical micelle concentrations of the surfactants, polymers and their mixtures were determined from surface tension versus concentration (ln C) plots (Figs. 1 and 2). The CMCs values of the pure systems were agreed well with literature values ${ }^{25-28}$ (Table-1). Whereas the CMCs values obtained for mixed systems are listed

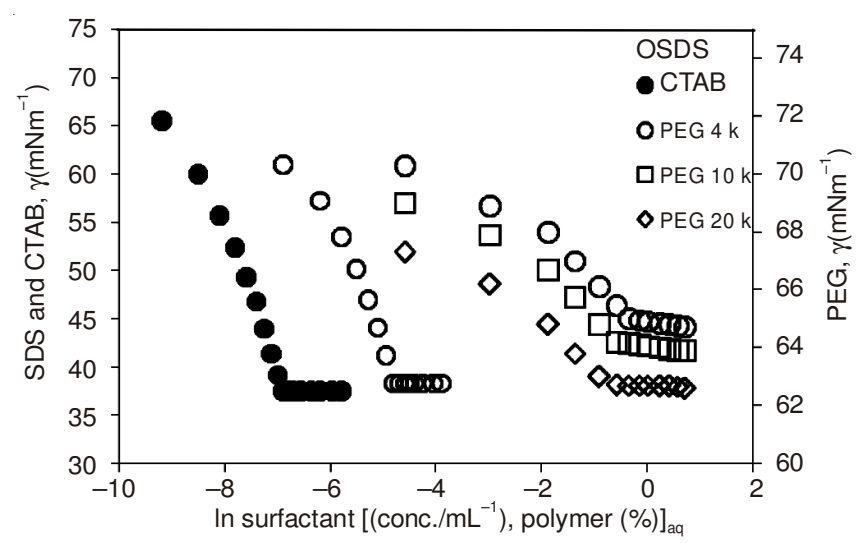

Fig. 1. Surface tension vs. In concentration of aqueous solution of surfactants and polymer alone

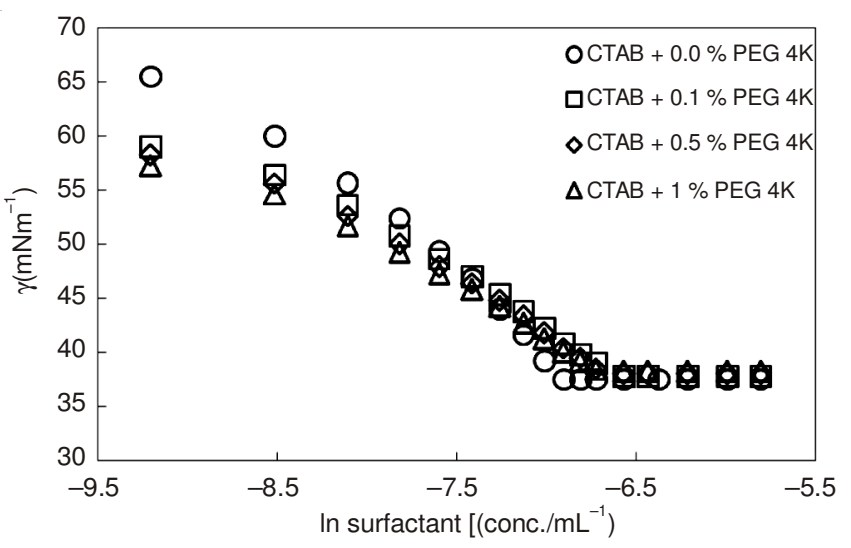

Fig. 2. Surface tension curve of CTAB and their mixture with PEG at $4 \mathrm{~K}$ $(0-1 \%)$

TABLE-1

CRITICAL MICELLAR CONCENTRATION (CMC), SURFACE EXCESSES CONCENTRATION, AREA PER MOLECULES, FREE ENERGY OF MICELLIZATION AND INTERFACIAL ADSORPTION ENERGY OF POLYETHYLENE GLYCOLE CALCULATED FROM SURFACE TENSION TECHNIQUE

\begin{tabular}{cccccc}
\hline PEG & $\begin{array}{c}\mathrm{CMC} \\
(\mathrm{mM})\end{array}$ & $\begin{array}{c}\Gamma_{\max } / \text { molecule } \\
(\mathrm{nm})\end{array}$ & $\begin{array}{c}\mathrm{A}_{\min } \\
\left(\mathrm{nm}^{2}\right)\end{array}$ & $\begin{array}{c}\Delta \mathrm{G}_{\text {mic }} \\
\left(\mathrm{kJ} \mathrm{mol}^{-1}\right)\end{array}$ & $\begin{array}{c}\Delta \mathrm{G}_{\text {ads }} \\
\left(\mathrm{kJ} \mathrm{mol}^{-1}\right)\end{array}$ \\
\hline $4 \mathrm{~K}$ & 0.017 & 0.24 & 4.22 & -37.20 & -55.12 \\
$10 \mathrm{~K}$ & 0.005 & 0.23 & 4.26 & -40.05 & -61.27 \\
$20 \mathrm{~K}$ & 0.002 & 0.22 & 4.47 & -42.28 & -68.63 \\
\hline
\end{tabular}

in Table-2. From these calculated CMCs of the surfactants and polymer, it is clear that CTAB is more surface active than SDS while that of PEG of higher molecular weight shows more surface activity compare to its lower weight PEG i.e. the CMC of PEG $20 \mathrm{~kg} / \mathrm{mol}>$ PEG $10 \mathrm{~kg} / \mathrm{mol}>4 \mathrm{~kg} / \mathrm{mol}$. The surface activity of PEG shows the only $20 \%$ mass of the polymer anchored to the interface whereas the remaining mass of the polymer form long bulk phase loops ${ }^{29}$. All the curves of surface tension of pure surfactant and its mixture with PEG are same as observed by others for such systems ${ }^{26,30}$. The plot of surface tension $v s$. concentration of pure surfactant or their mixture with polymer can be divided into three regions. Region I, starts from very dilute concentration where surfactant molecules are present in molecular state in solution to a point called as critical concentration represented as CAC or T1 (pure surfactant and mixture respectively) where surfactants molecules coexist in 
TABLE-2

CRITICAL MICELLAR CONCENTRATION (CMC), SURFACE EXCESSES CONCENTRATION, AREA PER MOLECULES, FREE ENERGY OF MICELLIZATION, INTERFACIAL ADSORPTION ENERGY AND COUNTER ION BOUND OF CTAB AND SDS WITH AND WITHOUT PEG, CALCULATED FROM SURFACE TENSION AND CONDUCTANCE TECHNIQUES

\begin{tabular}{|c|c|c|c|c|c|c|c|c|}
\hline & Surfactants + PEG & $\begin{array}{c}\mathrm{CMC}_{\text {Cond }} \\
(\mathrm{mM})\end{array}$ & $\begin{array}{c}\mathrm{CMC}_{\mathrm{S} . \mathrm{T}} \\
(\mathrm{mM})\end{array}$ & $\begin{array}{c}\Gamma_{\max } / \text { molecule } \\
(\mathrm{nm})\end{array}$ & $\mathrm{A}_{\mathrm{cmc}}\left(\mathrm{nm}^{2}\right)$ & $\underset{\left(\mathrm{kJ} \mathrm{mol}^{-1}\right)}{\Delta \mathrm{G}_{\text {mic }}}$ & $\underset{\left(\mathrm{kJ} \mathrm{mol}^{-1}\right)}{\Delta \mathrm{G}_{\mathrm{ads}}}$ & $\begin{array}{c}\text { B } \\
\text { (No. unit) }\end{array}$ \\
\hline \multirow{10}{*}{ SDS } & SDS $(0 \%)+$ PEG $4 \mathrm{~K}$ & 8.05 & 8.15 & 1.94 & 0.51 & -21.90 & -32.50 & 0.59 \\
\hline & $\operatorname{SDS}(0.1 \%)+$ PEG $4 \mathrm{~K}$ & 7.25 & 7.30 & 1.55 & 0.64 & -22.14 & -34.80 & 0.45 \\
\hline & $\operatorname{SDS}(0.5 \%)+$ PEG $4 \mathrm{~K}$ & 7.05 & 7.15 & 1.41 & 0.71 & -22.20 & -36.02 & 0.43 \\
\hline & SDS $(1 \%)+$ PEG $4 \mathrm{~K}$ & 7.00 & 7.01 & 1.33 & 0.75 & -22.24 & -36.73 & 0.39 \\
\hline & SDS $(0.1 \%)+$ PEG $10 \mathrm{~K}$ & 6.60 & 6.74 & 1.44 & 0.73 & -22.34 & -35.69 & 0.38 \\
\hline & SDS $(0.5 \%)+$ PEG $10 \mathrm{~K}$ & 6.55 & 6.60 & 1.41 & 0.78 & -22.40 & -35.91 & 0.36 \\
\hline & $\operatorname{SDS}(1 \%)+$ PEG $10 \mathrm{~K}$ & 6.30 & 6.35 & 1.40 & 0.82 & -22.50 & -35.91 & 0.33 \\
\hline & SDS $(0.1 \%)+$ PEG $20 \mathrm{~K}$ & 6.10 & 6.10 & 1.18 & 0.84 & -22.59 & -38.49 & 0.34 \\
\hline & SDS $(0.5 \%)+$ PEG $20 \mathrm{~K}$ & 5.90 & 5.95 & 1.15 & 0.87 & -22.64 & -38.78 & 0.32 \\
\hline & SDS $(1 \%)+$ PEG $20 \mathrm{~K}$ & 5.80 & 5.74 & 1.09 & 0.91 & -22.74 & -39.53 & 0.30 \\
\hline \multirow{10}{*}{ CTAB } & CTAB $(0 \%)+$ PEG 4 K & 1.01 & 1.01 & 2.10 & 0.47 & -27.04 & -37.10 & 0.71 \\
\hline & CTAB $(0.1 \%)+$ PEG $4 \mathrm{~K}$ & 1.10 & 1.12 & 1.76 & 0.57 & -26.78 & -37.96 & 0.68 \\
\hline & CTAB $(0.5 \%)+$ PEG $4 \mathrm{~K}$ & 1.15 & 1.18 & 1.65 & 0.60 & -26.65 & -38.66 & 0.66 \\
\hline & CTAB $(1 \%)+$ PEG $4 \mathrm{~K}$ & 1.25 & 1.28 & 1.64 & 0.61 & -26.45 & 38.63 & 0.65 \\
\hline & CTAB $(0.1 \%)+$ PEG $10 \mathrm{~K}$ & 1.20 & 1.24 & 1.76 & 0.57 & -26.53 & -37.84 & 0.65 \\
\hline & CTAB $(0.5 \%)+$ PEG $10 \mathrm{~K}$ & 1.30 & 1.29 & 1.71 & 0.58 & -26.43 & -38.20 & 0.63 \\
\hline & CTAB $(1 \%)+$ PEG $10 \mathrm{~K}$ & 1.42 & 1.40 & 1.60 & 0.62 & -26.23 & -38.86 & 0.61 \\
\hline & $\operatorname{CTAB}(0.1 \%)+$ PEG $20 \mathrm{~K}$ & 1.40 & 1.36 & 1.64 & 0.61 & -26.30 & -38.59 & 0.62 \\
\hline & CTAB $(0.5 \%)+$ PEG $20 \mathrm{~K}$ & 1.50 & 1.45 & 1.61 & 0.62 & -26.14 & -38.69 & 0.60 \\
\hline & CTAB $(1 \%)+$ PEG $20 \mathrm{~K}$ & 1.60 & 1.55 & 1.57 & 0.64 & -25.98 & -39.01 & 0.59 \\
\hline
\end{tabular}

equilibrium with a monolayer at air water interface and start aggregate formation on the polymer chain . Region II, starts from CAC and end at T2 (in case of mixed system) or CMC (pure surfactant solution)where at T2 the surfactant micellefully saturate the polymer and the point of minimum surface tension called as critical micelle concentration $\mathrm{CMC}^{26,27}$. Region III, starts from CMC or T2 (surfactant alone and surfactant polymer mixture respectively) and in case of pure surfactant the curve remains straight after CMC due to no addition of surfactant molecules to the interface while in case of polymer surfactant mixture there is T3 point at which the surfactant molecules replace all the polymer molecules or its loop from the interface and the surface tension curves of the pure surfactant and polymer surfactant mixtures become level in most mixed system (CTAB-PEG studied). But in SDS-PEG, at T3 the surface tension curve not become level to SDS surface tension curve because of the presence of some loops of PEG at the interface due to ion dipole interaction between SDS and PEG. The addition of polymer (PEO $4 \mathrm{~kg} / \mathrm{mol}$ ) to surfactant solution changes the shape of surface tension curve due to many factors depending upon the nature of surfactant and polymer, among them hydrophobicity and surface activity play important role for the reactivity of ionic surfactant and neutral polymer ${ }^{31}$. From Fig. 2 it can be seen that surface tension curve of mixed system is lower than the pure surfactant curve in the dilute region due to the reason that polymer molecules or loops of polymer are also present on the interface along with surfactant molecules. As the concentration of the CTAB-PEG $4 \mathrm{~kg} / \mathrm{mol}$ increases the difference in the surface tension curve of the pure surfactant and surfactant + PEG become zero at point which is called as $\mathrm{T} 1$. In $\mathrm{T} 2$ region the higher surface tension curve compared to that of pure surfactants indicates that there present mixed aggregates of polymer with surfactant. Further increases in concentration of the mix system, the gradual replacement of the polymer takes place which can be seen from the curve difference of the mix system and that of the pure surfactant becomes nearly zero at point T3. Further increase in concentration of surfactant in CTAB-PEG $(4 \mathrm{~kg} /$ mol) may result in changing the aggregate size and shape ${ }^{25}$ rather than changing the surface tension. From Fig. 2 it can be noted that as the concentration of PEG $4 \mathrm{~K}$ increases from 0.1 to $1 \%$ the difference in the surface tension curves of pure surfactant CTAB and CTAB-PEG mixture increases, (T1 decreases and T3 increases). From the intersection point of the curves in Fig. 2 the CMCs of the aqueous solution of CTAB alone and its mixture with PEG 4K/0-1\% can be calculated which is listed in the Tables 1 and 2 respectively. Fig. 2 indicated that by the addition of PEG to CTAB, the CMC of the CTAB increases and this effect is more pronounced if the concentration of the polymer is increased from $0.1-1 \%$.

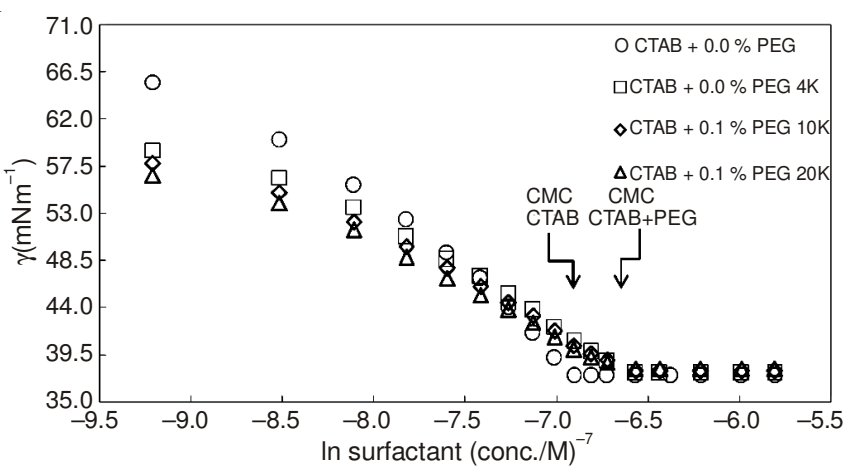

Fig. 3. Surface tension curves of CTAB alone and their mixture with $0.1 \%$ PEG 4, 10 and $20 \mathrm{~kg} / \mathrm{mol}$

Fig. 4 shows the effect of PEG (4 kg/mol, concentration effect) over the surface tension of SDS. The surface tension curve of SDS pure and its mixture with PEG are in general alike to the CTAB and its mixture with PEG but with some difference in the $\mathrm{CMC}$ region and $\mathrm{T} 3$ point which are the results 


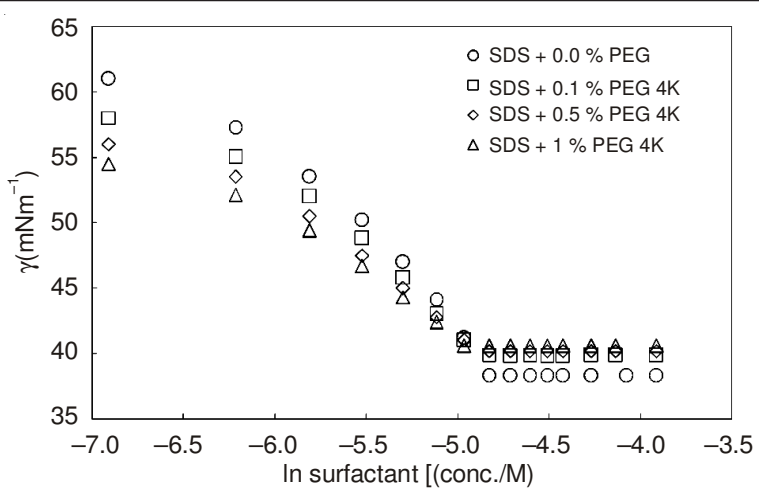

Fig. 4. Effect of (0-1 \%) PEG $4 \mathrm{~kg} / \mathrm{mol}$ the over the CMC of SDS

of different interaction of polymer with SDS. Fig. 4 also shows the lower surface tension curve for SDS-PEG mixture in the dilute region than the curve of the pure SDS due to associative behaviour of the mixed system. It can also be seen from the Fig. 4 that competitive adsorption of molecules or loops of PEG and surfactant molecules for interface start and free PEG contents been displaced from the interface by surfactant molecules at T1 point but here in SDS-PEG system the all the contents of PEG cannot be displaced from the interface which is clear from the synergetic surface tension curve (low in effectiveness of surface tension) of the mixed SDS-PEG system after T1. Furthermore the calculated CMC of the mixed system SDS-PEG is lower than the pure SDS as seen before ${ }^{25}$. Also with increase in concentration and molecular weight of the polymer of the mixed system decreases more the T1 and CMC. Quantitatively CMC of CTAB and SDS with respect to polymer concentration and molecular weight has been listed in the Table-2 but to zoom in the effect of concentration and molecular weight of PEGs over CMC of CTAB and SDS is shown in Figs. 6 and 7.

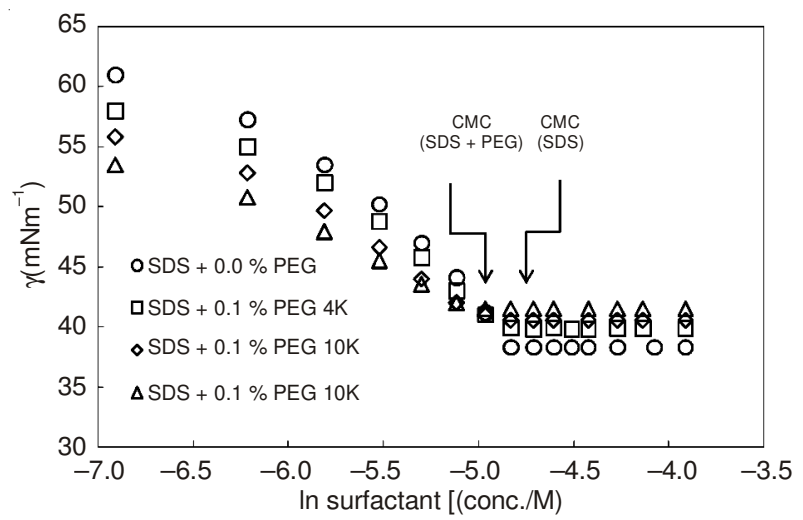

Fig. 5. Effect of $0.1 \%$ PEG 4, 10 and $20 \mathrm{~kg} / \mathrm{mol}$ over the CMC of SDS

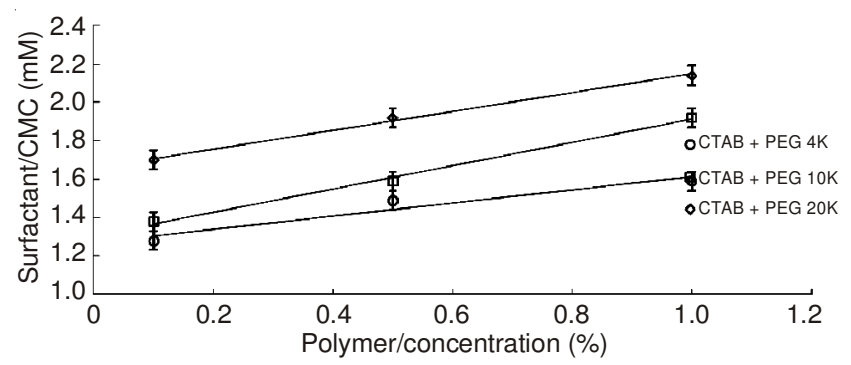

Fig. 6. Effect of (0-1\%) of PEG 4, 10 and $20 \mathrm{~kg} / \mathrm{mol}$ over the CMC of CTAB

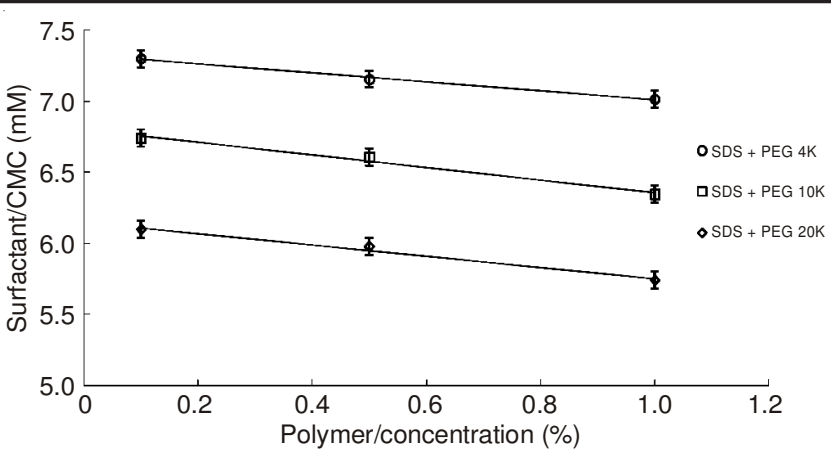

Fig. 7. Effect of (0-1\%) of PEG 4, 10 and $20 \mathrm{~kg} / \mathrm{mol}$ over the CMC of SDS

From the slope of the surface tension curve (linear line immediate before $\mathrm{CMC}$ ), surface excess concentration at the surface of the solution can be measured by using Gibbs farmalism $^{32,33}$.

$$
\Gamma_{\max }=-1 / \mathrm{RT}[\partial \gamma / \partial \operatorname{lnC}]_{\mathrm{T}}
$$

Here $\Gamma_{\max }$, is the surface excess concentration, $\mathrm{C}$ is the surfactant concentration, $\mathrm{R}$ gas constant and $\mathrm{T}$ is absolute temperature.

This parameter is helpful to understand the surface structure of the solution; values of this parameter for all the systems (CTAB and SDS with and without PEG) have been calculated using eqn. 1 are listed in the Table-2. $\Gamma_{\max }$ values of CTAB and SDS calculated by using equation are 2.10 and 1.94 molecules/ $\mathrm{nm}^{2}$, respectively are in close agreement with the literature ${ }^{33-35}$. The surface excess concentration of CTAB decreases to 1.76 molecule $/ \mathrm{nm}^{2}$ when $0.1 \%$ PEG $4 \mathrm{~kg} / \mathrm{mol}$ is added to CTAB solution further more when the concentration is increased to 1 $\%$ and molar mass to $20 \mathrm{~kg} / \mathrm{mol}$, surface excess concentration of CTAB decreased to 1.57 molecules $/ \mathrm{nm}^{2}$. The effect of PEG over the $\Gamma_{\max }$ of CTAB is accordance to the literature ${ }^{33}$. This decrease in the said properties of CTAB may be due to the presence of the some segments of PEG at interface near CMC or due to the hydrophobic interaction between the CTAB and PEG in the bulk of the solution allowing lesser molecules of CTAB to come at the interface in the presence of PEG rather than in the absence of PEG (at T3 point all the polymer segment have been completely removed from the interface by CTAB molecules). The effect of PEG over the $\Gamma_{\max }$ of SDS is also in same direction as that of CTAB, decrease in $\Gamma_{\max }$ of SDS with PEG addition was reported earlier ${ }^{36}$ but greater compared to CTAB. $\Gamma_{\max }$ of SDS decrease to 1.55 molecules $/ \mathrm{nm}^{2}$ when $0.1 \%$ of PEG $4 \mathrm{~kg} / \mathrm{mol}$ added and when the concentration is increased to $1 \%$ and molar mass to $20 \mathrm{~kg} / \mathrm{mol} \Gamma_{\max }$ of SDS decrease farther to1.10 molecules $/ \mathrm{nm}^{2}$. Here the decrease in the $\Gamma_{\max }$ of SDS is greater compared to CTAB with the addition of PEG and this is due to the strong ion diploe interaction at solution interface (between the sulphate group of the SDS with dipole formed on oxygen and carbon atoms of PEG due to the different electronegativity of oxygen and carbon atoms) compared to the weak hydrophobic interaction between CTAB and PEG molecules (in the bulk not at interface of solution). From the surface excess concentration, another important parameter i.e., minimum area per molecule $\left(\mathrm{A}_{\min }\right)$ can be calculated by using equation ${ }^{36}$. 


$$
\mathrm{A}_{\min }=1 \times 10^{18} / \mathrm{N}_{\mathrm{A}} \Gamma_{\max }
$$

where $\Gamma_{\max }$ is the surface excess concentration and $\mathrm{N}_{\mathrm{A}}$ is avogadro number.

Using eqn. 2, $\mathrm{A}_{\min }$ for all the systems i.e., CTAB and SDS with and without PEG have been calculated and are listed in Table-2. $A_{\min }$ of CTAB and SDS are 0.48 and $0.51 \mathrm{~nm}^{2}$ reported in the literature ${ }^{33,35}$. Addition of $0.1 \% 4 \mathrm{~kg} / \mathrm{mol}$ of PEG to CTAB increase the $A_{\min }$ to $0.57 \mathrm{~nm}^{2}$ and when the concentration is increased from 0.1 to $1 \%$ and molar mass of PEG is increased from 4 to $20 \mathrm{~kg} / \mathrm{mol}, \mathrm{A}_{\min }$ of CTAB is further increase to $0.63 \mathrm{~nm}^{2}$. Increase in $A_{\min }$ of CTAB with addition of PEG have been reported earlier ${ }^{33}$. This small change in area per molecule of CTAB in the presence of PEG is due to the presence of some polymer segments present at the interface with surfactant molecules near CMC or the availability of lesser surfactant molecules to adsorb at interface due to hydrophobic interaction between CTAB and PEG in the bulk of solution. The effect of PEG over the $\mathrm{A}_{\text {min }}$ of SDS is very high and it near become double when concentration is increased from 0.1-1 $\%$ and molar mass of PEG is increased from $4-20 \mathrm{~kg} / \mathrm{mole}$. Literature support the change in $\mathrm{A}_{\min }$ of SDS with the addition of $\mathrm{PEG}^{35}$. Increase in $\mathrm{A}_{\min }$ of SDS with the addition of PEG show that there are lesser number of surfactant molecules adsorb at the air-water interface due the presence of PEG segments at the interface with SDS molecules and it is due to the strong interaction (ion-dipole) between SDS and PEG molecules.

Thermodynamic parameters of micellization and interfacial adsorption: Once the CMC of CTAB and SDS without and with respect to PEG (different concentration and molecular weight) have been calculated, thermodynamics parameters i.e., free energy of micellization can be calculated using the Gibbs equation.

$$
\Delta \mathrm{G}_{\mathrm{mic}}=\mathrm{RT} \ln \mathrm{X}_{\mathrm{cmc}}
$$

where $\mathrm{R}$ is gas constant, $\mathrm{T}$ is absolute temperature and $\mathrm{X}_{\mathrm{cmc}}$ is mole fraction of solution.

All the values $\Delta \mathrm{G}_{\text {mic }}$ for CTAB, SDS alone, CTAB-PEG and SDS-PEG have been obtained by using eqn. 3 are listed in Table-2. $\Delta \mathrm{G}_{\mathrm{mic}}$ of CTAB is $-27.04 \mathrm{~kJ} / \mathrm{mol}$ and is accordance to the literature ${ }^{37}$, increasing to $-26.78 \mathrm{~kJ} /$ mole with addition of $0.1 \%$ of $4 \mathrm{~kg} / \mathrm{mol}$ PEG. When the polymer molar mass is increased from 4 to $20 \mathrm{~kg} / \mathrm{mol}$ and concentration is increased to $1 \%$ than the value of $\Delta \mathrm{G}_{\text {mic }}$ is decreased to $-25.97 \mathrm{~kJ} / \mathrm{mol}$. The negative values of $\Delta \mathrm{G}_{\text {mic }}$ show the spontaneity of micellization of CTAB and with the addition of PEG to CTAB solution the decrease in the negative values of $\Delta \mathrm{G}_{\text {mic }}$ reveal the spontaneity of micellization been suppressed. As with the increase in molar mass and concentration of PEG change more the negative values of $\Delta \mathrm{G}_{\text {mic }}$, affecting more the spontaneity of micellization of CTAB. It is due to hydrophobic interaction of CTAB with PEG molecules in the bulk of the solution, complex aggregate of CTAB and PEGs form cooperatively therefore less molecules of surfactant are available to saturate the interface in the presence of polymer rather than in the absence of polymer. Similarly, when concentration increases or polymer molar mass increase more surfactant molecules are needed to interact in the bulk and less molecules come to the surface hence the
$\mathrm{CMC}$ and free energy of micellization increase. To visualize the effect of PEGs over $\Delta \mathrm{G}_{\text {mic }}$ of CTAB, Figs. 8 and 9 are included. At CMC the interface is saturated by CTAB and some PEG segments may be present with CTAB molecules but after CMC surfactant molecules compete the polymer segments at interface and at $\mathrm{T} 3$ point all PEG molecules or segments are displaced from the value of $\Delta \mathrm{G}_{\text {mic }}$ of SDS is $-21.90 \mathrm{~kJ} / \mathrm{mol}$ which is according to the literature ${ }^{38}$. With the addition of $0.1 \%$ PEG $4 \mathrm{~kg} / \mathrm{mol}$ the values of $-\Delta \mathrm{G}_{\text {mic }}$ increase to $22.14 \mathrm{~kg} / \mathrm{mol}$ and with increasing concentration to $1 \%$ and molar mass of PEG to $20 \mathrm{~kg} / \mathrm{mol}$ than the value of $-\Delta \mathrm{G}_{\text {mic }}$ of SDS increase to $22.74 \mathrm{~kJ} / \mathrm{mol}$ which show that the spontaneity of SDS micellization increases with the addition of PEG. This opposite behaviour of SDS in the presence of PEG can be explained by the strong ionic dipole type of interaction (anionic head of the surfactant/SDS and the dipole produced by the difference in the electronegativity between oxygen atom and carbon atom in PEG chain) at the interface residing some of the PEG molecules to the surface even near $\mathrm{CMC}^{39}$ increasing the spontaneity of micellization. Here it can be said that with the polymer concentration increase, free energy of micellization decreases which mean that surfactant molecules give favor to more polymer molecules or loop of polymer to reside at the interface with surfactant molecules through the ionic dipole type of interaction, reducing free energy of micellization of SDS.

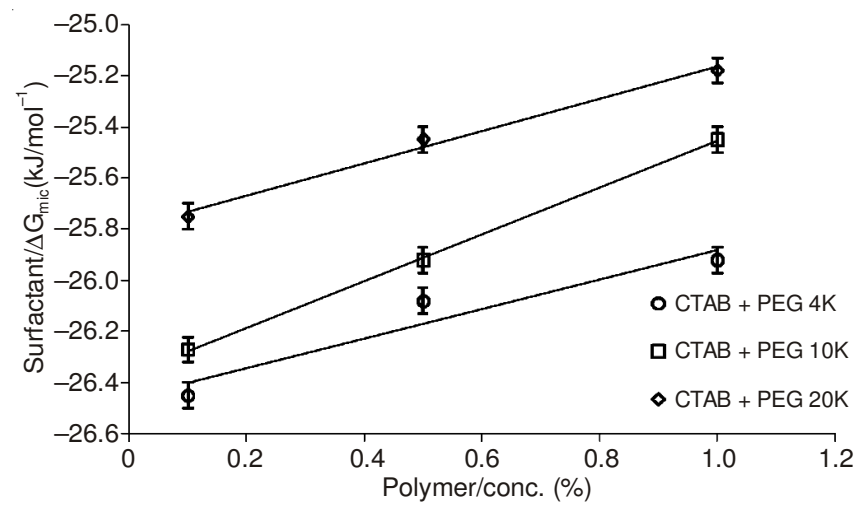

Fig. 8. Shows the effect of (0.1-1\%) of PEG (4, 10 and $20 \mathrm{~kg} / \mathrm{mol})$ over the free energy of micellization of CTAB

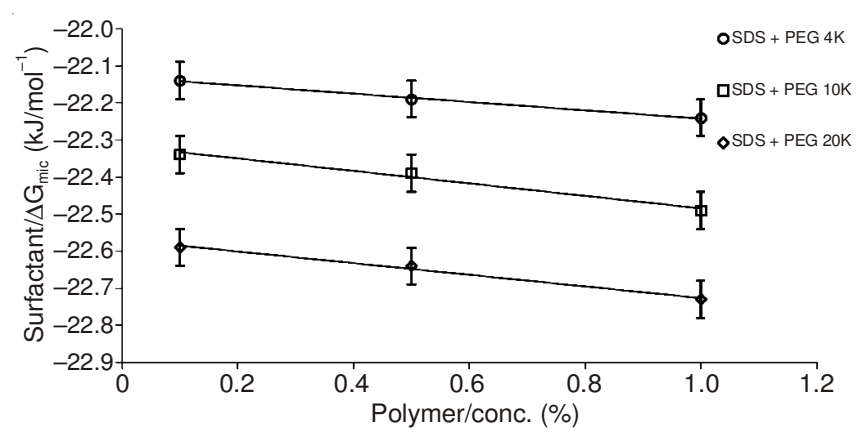

Fig. 9. Shows the effect of (0.1-1\%) of PEG (4, 10 and $20 \mathrm{~kg} / \mathrm{mol})$ over the free energy of micellization of SDS

Free energy of adsorption at air water interface $\left(\Delta \mathbf{G}_{\mathrm{ads}}\right)$ : Free energy of adsorption is an important parameter to know about the surfactant adsorption at air water interface of aqueous solution of surfactant,can be calculated from equation ${ }^{40}$. 


$$
\Delta \mathrm{G}_{\mathrm{ads}}=\Delta \mathrm{G}_{\mathrm{mic}}-\pi_{\mathrm{cmc}} / \Gamma_{\max }
$$

where $\Delta \mathrm{G}_{\text {ads }}$ is change in free energy of interfacial adsorption, $\Delta \mathrm{G}_{\mathrm{mic}}$ change in free energy of micellization at CMC, $\pi_{\mathrm{cmc}}$ is the surface pressure at $\mathrm{CMC}$ and $\Gamma_{\max }$ is the maximum surface excess concentration. $\pi_{\mathrm{cmc}}$ values for the aqueous solution of surfactants alone and their mixed aqueous solution with PEG lying in the range of 30.5 to $34.5 \mathrm{mN} / \mathrm{m}$ which is according to literature ${ }^{33}$. Values of $\Delta \mathrm{G}_{\text {ads }}$ calculated using eqn. 4 are -32.50 , $-37.10,-55.12,-61.27$ and $-68.63 \mathrm{~kJ} / \mathrm{mol}$ for SDS, CTAB at 4, 10 and $20 \mathrm{~K}$, listed in Tables-2 and 1, respectively. Mixing PEG with SDS and CTAB change the $\Delta \mathrm{G}_{\mathrm{ads}}$ of surfactant. $-\Delta \mathrm{G}_{\mathrm{ads}}$ of SDS increase with the addition of PEG and increase when concentration or molar mass of PEG increase. $-\Delta \mathrm{G}_{\text {ads }}$ of CTAB also increase with the addition of PEG but here the effect of PEG over $\Delta \mathrm{G}_{\mathrm{ads}}$ of CTAB is lesser compared to SDS which show strong interaction between SDS and PEG at interface compared to CTAB and PEG interaction as result adsorption of CTAB is effected less in the presence of PEG as compared to SDS.

Variation of fraction of counter ion binding $(\boldsymbol{\beta})$ : Conductance measurement for the above systems also gives valuable information about the CMC and fraction of counter ion binding to the micelle formation. Fraction of counter ion binding $(\beta)$ is important parameter in micelle formation. Two types of opposing forces are responsible for micellization of ionic surfactant: (a) Favors micelle formation i.e., responsible for the removal of hydrocarbon chain from water matrix (b) Disfavors micelle formation i.e., arises due to the electrostatic repulsion among the similar charged head groups. Measurement of counter ions bound $(\beta)$ to the micellar interface is basically decreasing the opposing force of charged head groups and increasing the favor of micelle formation ${ }^{41,42}$. Counter ion bound can be obtained from degree of micelle ionization (a) i.e., $\beta=(1-\alpha)$, while degree of micelle ionization is obtained from the ratio of the post micellar slope (S2) to the pre micellar slope (S1) from the conductance vs. concentration curve of the surfactant.

$$
\alpha=\mathrm{S} 2 / \mathrm{S} 1
$$

Figs. 10 and 11 show conductance $v s$. concentration curve of CTAB and SDS with and without $(0.1 \%$ of 4,10 and 20 $\mathrm{kg} / \mathrm{mol}$ ), respectively. All the values of the counter ions bound are listed in the Table- 1 for all the systems of CTAB and SDS with PEG (different concentration and molar mass). $\beta$ values obtained for aqueous solution of SDS and CTAB are 0.593 and 0.71 , respectively are according to the earlier reported literature values ${ }^{33,42,43}$. $\beta$ value decreases with the addition of PEG to the aqueous solution of SDS, also with increase in concentration and molar mass of PEG decrease the $\beta$ as reported $^{35}$ which mean that micellization of SDS is enhanced and actually with the addition of PEG, CMC of SDS decreases as observed here and by other ${ }^{25}$. Therefore this decrease in $\beta$ is due to the ion dipole interaction between SDS and PEG molecules which shield the electrostatic repulsion between head group of SDS. A consistent increase in the $\beta$ value is observed here for the mixed system of CTAB-PEG (with increase in concentration and molar mass of PEG). Decrease in $\beta$ shows that $\mathrm{CMC}$ of $\mathrm{CTAB}$ will decrease with the addition of PEG but actually increase in CMC is observed here and is

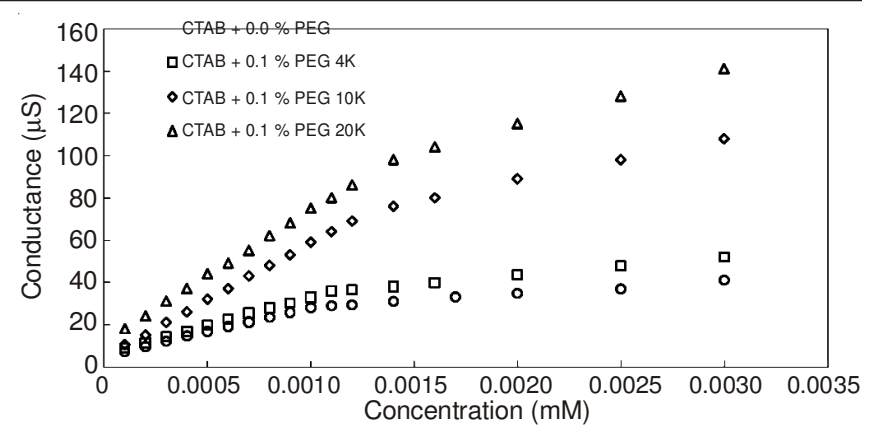

Fig. 10. Conductance vs. concentration of CTAB with and without $0.1 \%$ PEG of 4, 10 and $20 \mathrm{~kg} / \mathrm{mol}$

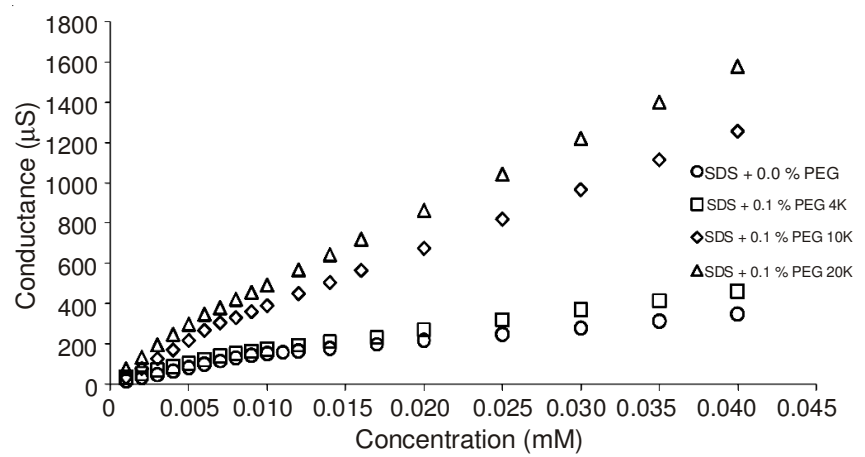

Fig. 11. Conductance vs. concentration of SDS with and without $0.1 \%$ PEG of 4,10 and $20 \mathrm{~kg} / \mathrm{mol}$

accordance to the literature ${ }^{28}$. This anomalous behaviour (CMC increases and $\beta$ decreases) is because of hydrophobic interaction is dominant over the ion dipole interaction between the ionic head of surfactant molecules to the dipole formed between the oxygen atom and carbon atom with in the PEG chain due electronegative difference between them which shield the electrostatic repulsion of the head group of CTAB enhancing micellization however the hydrophobic interaction between CTAB and PEG do stay some of the CTAB molecules in the bulk of the solution. Due to this reason decrease in $\beta$ do not affect the increase in CMC of CTAB.

Counter ion bound to the micelle surface against polymer concentration for the two surfactants can be graphically represented in Figs. 12 and 13.

\section{Conclusion}

A systematic study on the effect of concentration and molar mass of PEG over the interfacial adsorption, micellization and associated parameters of CTAB and SDS have been evaluated in this report, which helped to find out the factors responsible for the different behaviour of CTAB and SDS in presence of PEG. Interfacial adsorption of CTAB in the presence of PEG is reduced while that of SDS interfacial adsorption has been enhanced in the presence of PEG. The effect in above said property of CTAB and SDS is more pronounce when the concentration or molar mass of PEG increases. Critical micelle concentration of CTAB increases in the presence of PEG because of the hydrophobic interaction between CTAB and PEG in the bulk of the solution, need more molecules of CTAB to saturate the interface to get CMC but the CMC of SDS decrease in the presence of PEG. This is due to the ion dipole interfacial interaction between the said components of the solution. The change in the CMC of CTAB and SDS 


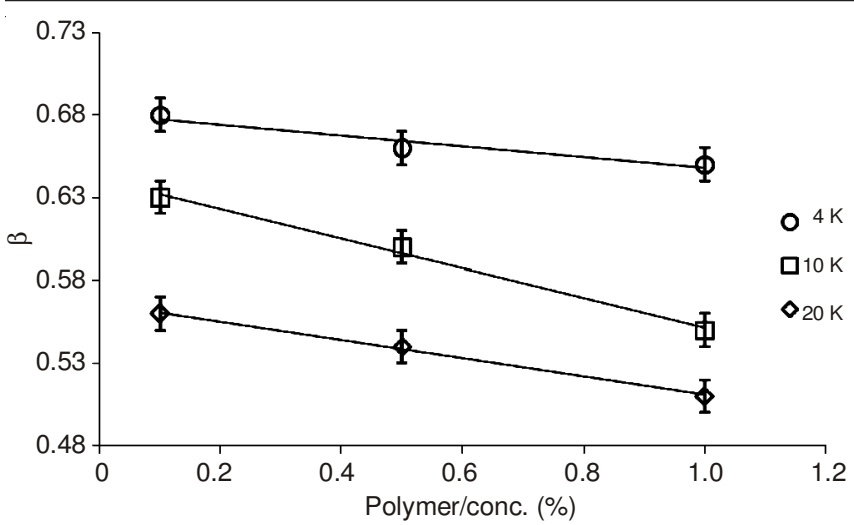

Fig. 12. Counter ion bounds of CTAB vs. polymer concentration $(4,10,20$ $\mathrm{kg} / \mathrm{mol})$

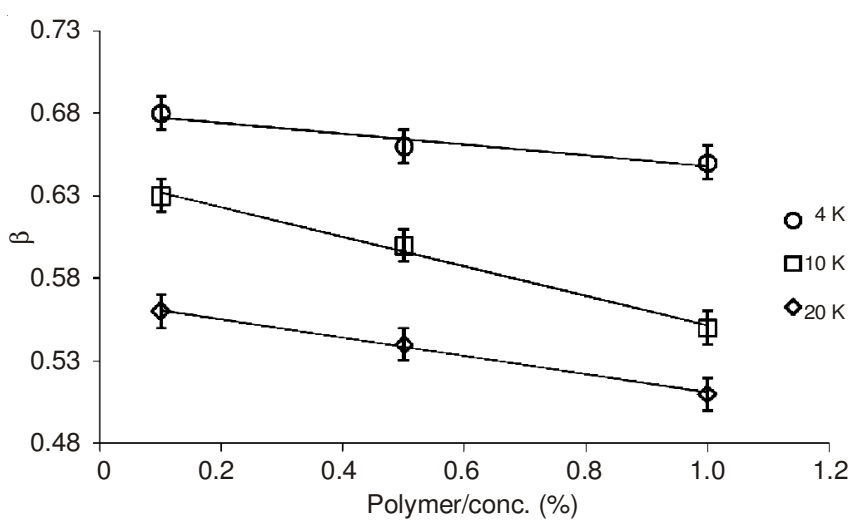

Fig. 13. Counter ion bounds of SDS vs. polymer concentration (4, 10, 20 $\mathrm{kg} / \mathrm{mol})$

increase as the concentration and molar mass of PEG increases. Thermodynamic parameters calculated for CTAB-PEG and SDS-PEG systems, strengthen the above conclusions. Counter ions bound of the two surfactants decrease in the presence of PEG which favors the decrease in the CMC of SDS but need explanation as CMC of CTAB increase with the addition of PEG. Finally it was concluded that PEG interact hydrophobically with CTAB molecules in the bulk of solution and do not interact at the interface, all the PEG segments been displaced by CTAB molecules from the interface near in the $\mathrm{CMC}$ region. While ion dipole type of interaction is present between SDS and PEG segments at the interface with additional hydrophobic interaction in the bulk of solution.

\section{REFERENCES}

1. E. Goddard and J. Gruber, Principles of Polymer Science and Technology in Cosmetics and Personal Care, Dekker: New York (1999).

2. B. Cabane, J. Phys. Chem., 81, 1639 (1977).

3. E. Goddard and A. Ananthapadmanabhan, Interactions of Surfactants with Polymers and Proteins. CRC Press: Boca Raton, FL (1993).

4. L.-T. Lee, Curr. Opin. Colloid Interface Sci., 4, 205 (1999).

5. F.E. Antunes, E.F. Marques, M.G. Miguel and B. Lindman, Adv. Colloid Interface Sci., 147, 18 (2009).

6. R. López-Esparza, M.-A. Guedeau-Boudeville, Y. Gambin, C. Rodríguez-Beas, A. Maldonado and W. Urbach, J. Colloid Interf. Sci., 300, 105 (2006).
7. K. Holmberg, B. Jönsson, B. Kronberg and B. Lindman, Surfactants and Polymers in Aqueous Solution, Wiley: New York (1998).

8. A.A. Ruzza, S.J. Froehner, E. Minatti, F. Nome and D. Zanette, J. Phys. Chem., 98, 12361 (1994).

9. M.N. Jones, J. Colloid Interf. Sci., 23, 36 (1967).

10. E.D. Goddard and R.B. Hannan, J. Colloid Interf. Sci., 55, 73 (1976).

11. J. Phys. Chem., 75, 2740 (1975).

12. D. Zanette and V.L.A. Frescura, J. Colloid Interf. Sci., 213, 379 (1999).

13. E.D. Goddard and P.S. Leung, Langmuir, 8, 1499 (1992).

14. D.P. Norwood, E. Minatti and W.F. Reed, Macromolecules, 31, 2957 (1998).

15. K. Chari and W.C. Lenhart, J. Colloid Interf. Sci., 137, 204 (1990).

16. T.P. Goloub, R.J. Pugh and B.V. Zhmud, J. Colloid Interf. Sci., 229, 72 (2000).

17. Y.-Q. Zhang, T. Tanaka and M. Shibayama, Super-Absorbency and Phase Transition of Gels in Physiological Salt Solutions (1992).

18. D. Süss, Y. Cohen and Y. Talmon, Polymer, 36, 1809 (1995).

19. M.I. Gjerde, W. Nerdal and H. Høiland, J. Colloid Interf. Sci., 197, 191 (1998).

20. J. Colloid Interface Sci., 184, 260 (1994).

21. F.M. Winnik and S.T.A. Regismond, Colloids Surf. A, 118, 1 (1996).

22. C.F. Lima, F. Nome and D. Zanette, J. Colloid Interf. Sci., 187, 396 (1997).

23. D. Zanette, Ã.A. Ruzza, S.J. Froehner and E. Minatti, Colloids Surf. A, 108, 91 (1996).

24. T. Vongsetskul, D.J.F. Taylor, J. Zhang, P.X. Li, R.K. Thomas and J. Penfold, Langmuir, 25, 4027 (2009).

25. M.K. Baloch, F. Ahmad, A. Rauf and G.F. Durrani, J. Appl. Polym. Sci., 114, 1444 (2009).

26. J. Penfold, R.K. Thomas, X.L. Zhang and D.J.F. Taylor, Langmuir, 25, 3972 (2009).

27. D.J.F. Taylor, R.K. Thomas, J.D. Hines, K. Humphreys and J. Penfold, Langmuir, 18, 9783 (2002).

28. M.K. Baloch, A. Rauf, F. Ahmad, G.F. Durani and G. Hameed, J. Appl. Polym. Sci., 116, 2133 (2010).

29. M. Darvas, T. Gilányi and P. Jedlovszky, J. Phys. Chem. B, 114, 10995 (2010).

30. T. Gilányi, I. Varga, M. Gilányi and R. Mészáros, J. Colloid Interf. Sci., 301, 428 (2006).

31. M. Darvas, T. Gilányi and P. Jedlovszky, J. Phys. Chem. B, 115, 933 (2011).

32. M.J. Rosen, Emulsification by Surfactants. Surfactants and Interfacial Phenomena, edn. 3, pp. 303-331 (2004).

33. K. Manna and A.K. Panda, J. Surfact. Deter., 14, 563 (2011).

34. H.B. de Aguiar, M.L. Strader, A.G.F. de Beer and S. Roke, J. Phys. Chem. B, 115, 2970 (2011).

35. A.K. Panda, G. Sarkar and K. Manna, J. Disp. Sci. Technol., 30, 1152 (2009).

36. T. Chakraborty, S. Ghosh and S.P. Moulik, J. Phys. Chem. B, 109, 14813 (2005).

37. B.L. Bales and R. Zana, Langmuir, 20, 1579 (2004)

38. N. Dubey, J. Surf. Sci. Technol., 24, 139 (2008).

39. D.J. Cooke, C.C. Dong, J.R. Lu, R.K. Thomas, E.A. Simister and J. Penfold, J. Phys. Chem. B, 102, 4912 (1998).

40. L. Bernazzani, R. Carosi, P. Gianni and V. Mollica, J. Solution Chem., 38, 1369 (2009).

41. N. Jiang, P. Li, Y. Wang, J. Wang, H. Yan and R.K. Thomas, J. Colloid Interf. Sci., 286, 755 (2005).

42. N.V. Lebedeva, A. Shahine and B.L. Bales, J. Phys. Chem. B, 109, 19806 (2005)

43. S.S. Shah, N.U. Jamroz and Q.M. Sharif, Colloids Surf. A, 178, 199 (2001). 